\title{
Readmissions of long-stay psychiatric patients to the acute services from hostels in the community
}

\author{
Rosie SHePPERD, Senior Registrar, Child and Adolescent Psychiatry, Oxford Rotation, \\ Paxton House, 57 Bath Road, Reading, Berkshire
}

The asylum movement was developed in the 19th century to provide care and cure for people with mental disorders. In the 20th century the old vision of asylum was abandoned, but no new alternative vision of community mental health care has taken its place. A divide between acute psychiatric services and provision for the social aspects of care has been described by Murphy (1991).

Friern Hospital is a large institution in North London serving Hampstead, Bloomsbury \& Islington, and West Haringey health authorities. It is due for closure in 1993 and to make provision for the needs of its patients a ten year plan was drawn up in 1983 which was facilitated by reprovision teams from the health authorities. As the policy of discharge to the community continued a number of patients, resident for over one year, were identified as being unable to live independently in the community at that time. Fifty-two such patients were from Hampstead Health Authority. Four properties were purchased within its catchment area for the continuing rehabilitation and accommodation of some of these patients.

The literature concerning reprovision for longstay patients has described better quality of life for new long-stay patients (Gibbons \& Butler, 1987); however, the observation is made that patients may feel more lonely and isolated, having lost a peer group and supportive community.

Health economists, Knapp et al (1990) examined the cost of community reprovision for the first 136 patients leaving Claybury and Friern Hospitals. Their predictions that the cost of community care would remain lower than hospital care for the full population of the two hospitals scheduled to close have not been substantiated. Hafner (1989) found the cost of community care exceeded the cost of hospital care for more disabled psychiatric patients. Research associated with the TAPS project has revealed that 22 patients $(6 \%)$ of Claybury and Friern's long-stay population were not successfully resettled in the community and an estimate of need for continuing care beds has been made by Dayson et al (1992).

The current study was designed to look at Hampstead Health Authority patients readmitted from these four hostels. Psychological assessment prior to discharge using the Adaptive Functioning Scale (Baker \& Hall, 1983) showed $80 \%$ of these patients to have severe or medium dependency. Two hostels were for the more elderly, non-demented population (age range 60-83). These provided a containing environment and are now managed by a Housing Association, Circle 33. Two hostels for the younger patients (age range 37-68) have more emphasis on continuing rehabilitation. All hostels are regularly visited by psychiatric staff and psychologists, who would provide urgent reassessments and daily telephone advice when necessary. Three out of the four hostels were allocated general practitioner services. Three hostels are staffed by psychiatric nurses, one hostel for the younger patients is managed by Camden Social Services and staffed by residential social workers. The hostels were opened at different times, Hostel A in 1986, B and C in 1988 and $D$ in 1989. They were refurbished to a high standard, with twin and single rooms, shared sitting rooms and gardens. There was less available supervised space than in the institution.

\section{The study}

A retrospective study was made of all patients discharged to these hostels until June 1990. A nonvalidated scoring system was used to provide a numerical assessment of level of functioning and symptomatology in hospital and in the hostels so comparisons could be made. A case-note study and interview with key members of staff was completed for each patient.

Psychiatric readmissions were to the Royal Free or Friern Hospitals, general medical admissions were to the Royal Free Hospital. A case-note search was used to count and group readmissions to the acute psychiatric services and general hospital from 1986 until June 1990.

\section{Findings}

Thirty-four patients were discharged to Hampstead Health Authority hostels from Friern Hospital during the study period. Because of small numbers the hostels were paired for the elderly and younger patients. There were 18 male and 16 female patients; 
the mean age of the elderly group was 73.7 years and 46.5 years in the younger group. The length of stay in hospital prior to discharge ranged from 1-60 years and mean length of stay was 20.8 years.

The diagnoses were 28 patients with schizophrenia, five with affective disorder and one with alcohol dependence syndrome. Four had additional diagnoses of moderate to severe learning difficulties.

The group means showed no significant improvement or deterioration after discharge, despite observed decline in some behaviour on relaxation of rehabilitation ward programmes. Two improved enough to be discharged to less supported accommodation. Twelve patients had readmissions (18 readmissions) to the acute psychiatric services until June 1990 . There were three deaths by natural causes from the elderly group. Seven patients had admissions to general hospitals, one patient having 26 admissions for alcohol induced hypoglycaemia, in an insulin dependent diabetic with alcohol dependence syndrome.

According to the $\chi^{2}$ analysis at $0.05 \%$ significance there were no significant differences in problem scores found between those readmitted and the whole group.

The reasons for readmissions included threatened violence to female staff; constant demands on staff eclipsing other patients' needs; deterioration in self care, not eating; amorous advances to a female resident; increased verbal responses to auditory hallucinations, with violent outbursts; relapse of depression with social withdrawal and self neglect; hypomanic relapse not responding to medication changes in the hostel; and auditory hallucinations, patient accused of killing neighbour's guinea pig. Only one readmission was known to be associated with noncompliance of medication.

In the first year of opening one of the younger hostels (D), three patients were readmitted; two of these had two readmissions and one had three. Two of these patients were not re-placed in the community. Hostel $A$ had no readmissions in the first year of opening, $B$ and $C$ had one readmission each.

\section{Comment}

Thirty-four patients from a very disabled group were discharged to Hampstead Health Authority hostel in the community from December 1986 to June 1990. There were 12 patients readmitted to the psychiatric services and seven to general hospital. Seven remained in-patients on the psychiatric wards at the end of the study period. Although those readmitted did not appear to be a more disabled group in total, the small numbers may have obscured statistically significant differences being shown.
Most of the readmissions were for deterioration in mental state and behavioural changes, including deteriorating self-care. There were threats of violence but only one episode of actual physical violence.

Hostel D had seven readmissions for three patients in its first year of opening, which was a higher rate than the other hostels. Being the last hostel to open these patients may have been a more disabled group. This hostel is managed by the London Borough of Camden and staffed by resident social workers; differences in training, psychiatric experience and philosophical approach from the other hostels may be relevant.

The continuing need for acute psychiatric beds to back up community care is clearly shown, and this group of patients may make a significant contribution to the use of acute beds. The future placement of the seven patients readmitted and not re-placed in the community is of interest, as will be the placement of patients found unsuitable for discharge to these hostels.

A follow-up survey of readmissions from this group since June 1990 would show whether this trend of readmissions has continued.

\section{Acknowledgements}

I would like to thank Dr Anne Bird, The Academic Department of Psychiatry, Royal Free Hospital, for advice and encouragement and Dr Donovan Hailstone and the Rehabilitation Unit, Friern Hospital for help in starting this project.

\section{References}

BAKER, R. \& HALl, J. N. (1983) Users Manual for Rehabilitation Evaluation. Scotland: Hall \& Baker, Vine Publishing.

Dayson, D., Gooch, C. \& Thornicroft, G. (1992) Difficult to place long term psychiatric patients: risk factors for failure to resettle long stay patients in community facilities. British Medical Journal, 305, 993-995.

Gibbons, J. S. \& BuTler, J. P. (1987) Quality of life for "new" long-stay psychiatric in-patients. The effects of moving into a hostel. British Journal of Psychiatry, 151, 347-354.

HAFNER, H. (1989) Effectiveness and cost of community care for schizophrenic patients. Hospital and Community Psychiatry, 40, 59.

Knapp, M., Beecham, J., Anderson, J., Dayson, D. et al (1990) Predicting the community costs of closing psychiatric hospitals. British Journal of Psychiatry, 157, 661-670.

MURPHY, E. (1991) Community mental health sevices; a vision for the future. British Medical Journal, 302, 1064-1065. 